\title{
Characteristics of and risk factors for biliary pathogen infection in patients with acute pancreatitis
}

Shayan Chen ${ }^{1,2}$, Jiyu Shi ${ }^{3}$, Minghui Chen ${ }^{1}$, Jun Ma' ${ }^{1}$ Zhaowei Zeng ${ }^{1}$, Rui Wang ${ }^{1}$, Yunfeng Cui ${ }^{4^{*}}$ and Xue Gao ${ }^{5^{*}}$

\begin{abstract}
Background: Infection in patients with acute pancreatitis, especially severe acute pancreatitis patients, is a common and important phenomenon, and the distributions and drug resistance profiles of bacteria causing biliary infection and related risk factors are dynamic. We conducted this study to explore the characteristics of and risk factors for bacterial infection in the biliary tract to understand antimicrobial susceptibility, promote the rational use of antibiotics, control multidrug-resistant bacterial infections and provide guidance for the treatment of acute pancreatitis caused by drug-resistant bacteria.

Methods: The distribution of 132 strains of biliary pathogenic bacteria in patients with acute pancreatitis from January 2016 to December 2020 were analyzed. We assessed drug resistance in the dominant Gram-negative bacteria and studied the drug resistance profiles of multidrug-resistant bacteria by classifying Enterobacteriaceae and nonfermentative bacteria. We then retrospectively analyzed the clinical data and risk factors associated with 72 strains of Gramnegative bacilli, which were divided into multidrug-resistant bacteria (50 cases) and non-multidrug-resistant bacteria (22 cases).
\end{abstract}

Results: The main bacteria were Escherichia coli, Acinetobacter baumannii, Klebsiella pneumoniae and Pseudomonas aeruginosa. Extended-spectrum beta-lactamase (ESBL)-producing Escherichia coli had a 66.67\% detection rate. Acinetobacter baumannii had more than 50.00\% drug resistance to carbapenems, ESBL-producing Klebsiella pneumoniae had $100.00 \%$ drug resistance, and Pseudomonas aeruginosa had $66.67 \%$ resistance to carbapenems. Multivariate logistic regression analysis suggested that the administration of third- or fourth-generation cephalosporins was an independent risk factor for Gram-negative multidrug-resistant biliary bacterial infection in acute pancreatitis patients.

Conclusion: Drug resistance among biliary pathogens in acute pancreatitis patients remains high; therefore, rational antimicrobial drug use and control measures should be carried out considering associated risk factors to improve diagnosis and treatment quality in acute pancreatitis patients.

Keywords: Acute pancreatitis, Biliary pathogens, Gram-negative bacteria, Multidrug-resistant bacteria, Risk factors

*Correspondence: nkyycyf@163.com; gxue0122@163.com

${ }^{4}$ Department of Surgery, Tianjin Medical University NanKai Hospital, 122

Sanwei Road, Nankai District, Tianjin 300100, China

${ }^{5}$ Binzhou Medical University, Yantai City 264003, Shandong, China

Full list of author information is available at the end of the article

\begin{abstract}
Background
Biliary infection in acute pancreatitis (AP) patients, especially severe acute pancreatitis (SAP) patients, is common and has been associated with invasive clinical procedures, septicemia, intestinal barrier dysfunction and gut bacteria translocation [1]. It remains an important cause of death in patients with SAP [2]; therefore, for
\end{abstract}

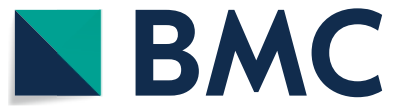

(c) The Author(s) 2021. Open Access This article is licensed under a Creative Commons Attribution 4.0 International License, which permits use, sharing, adaptation, distribution and reproduction in any medium or format, as long as you give appropriate credit to the original author(s) and the source, provide a link to the Creative Commons licence, and indicate if changes were made. The images or other third party material in this article are included in the article's Creative Commons licence, unless indicated otherwise in a credit line to the material. If material is not included in the article's Creative Commons licence and your intended use is not permitted by statutory regulation or exceeds the permitted use, you will need to obtain permission directly from the copyright holder. To view a copy of this licence, visit http://creativecommons.org/licenses/by/4.0/. The Creative Commons Public Domain Dedication waiver (http://creativeco $\mathrm{mmons}$.org/publicdomain/zero/1.0/) applies to the data made available in this article, unless otherwise stated in a credit line to the data. 
AP patients with bile infectious symptoms, bile samples are frequently used for clinical microbiological culture tests [3]. However, the bile bacterial spectrum and antibiotic resistance characteristics are constantly changing.

Gram-negative bacilli, which are some of the most common opportunistic pathogens in hospitals, have shown increasing trends towards drug resistance in recent years [4]. Gram-negative bacilli mainly include those in the Enterobacteriaceae family and nonfermentative bacteria. Nonfermentative bacteria often produce efflux pumps, induce position changes and enzyme production, develop various drug resistance mechanisms, and easily evolve into multidrug-resistant [5] organisms (MDROs) that are resistant to three or more types of clinical antibiotics used at the same time. In recent years, with the extensive use of antibiotics for the treatment of bacterial infections, MDROs have become common and important pathogens responsible for nosocomial infections, resulting in an intractable challenge for clinical diagnosis and treatment [6]; therefore, it is necessary to clarify the risk factors for MDRO infection in AP patients.

We conducted this study to understand the characteristics of pathogens causing biliary infections and related risk factors in patients with AP, guide clinical detection, promote the rational use of antibiotics, control multidrug-resistant bacterial infections, and improve the cure rate in AP patients. We analyzed the in-hospital data of AP patients from January 2016 to December 2020 to retrospectively identify the biliary pathogenic spectrum and drug resistance profiles, Gram-negative multidrug-resistant bacteria distributions and resistance rates, and associations of risk factors in the clinical characteristics of the patients. The study results are as follows.

\section{Results}

\section{General characteristics of the distributions} and proportions of bile pathogenic bacteria

A total of 120 positive cases were diagnosed and 132 pathogenic bacterial strains were isolated from 560 bile samples from AP patients. Duplicate strains from the same patient were excluded. The proportion of samples with more than one pathogen was $10.00 \%$. Seventy-two Gram-negative bacterial strains accounted for $54.55 \%$ of the isolates, 56 Gram-positive bacterial strains accounted for $42.42 \%$ of the isolates, and 4 fungi accounted for only 3.03\% of the isolates. Regarding Gram-negative bacteria, here mainly referred with Gram-negative bacilli, namely the Enterobacteriaceae family and nonfermentative bacteria. The Enterobacteriaceae family included Escherichia coli and Klebsiella pneumoniae, and so on, nonfermentative bacteria covered Acinetobacter baumannii and Pseudomonas aeruginosa, and so on. Fifty strains belonging to Enterobacteriaceae accounted for $37.88 \%$, and 22 nonfermentative bacterial strains accounted for $16.67 \%$. According to the drug resistance profiles, the percentage of multidrug-resistant bacterial strains with at least $50.00 \%$ resistance was $37.88 \%$; among them, 32 Enterobacteriaceae had at least $50.00 \%$ resistance, accounting for $24.24 \%$ of the total or $64.00 \%$ of the 50 Enterobacteriaceae strains isolated. Eighteen nonfermentative strains were multidrug resistant, accounting for $13.64 \%$ of the total or $81.82 \%$ of 22 strains of nonfermentative bacteria isolated. The distributions and proportions of pathogenic bacteria are shown in detail in Fig. 1A/B.

\section{Analysis of the drug resistance rates of Gram-negative pathogenic bacteria in the bile of AP patients}

In this study, the Gram-negative bacterium accounting for the largest proportion was Escherichia coli, followed by Acinetobacter baumannii, Klebsiella pneumoniae and Pseudomonas aeruginosa. The specific drug resistance rates are shown in Fig. 2.

\section{Resistance analysis of Gram-negative MDROs from bile samples from AP patients}

Resistance of Gram-negative MDROs was further analyzed by Enterobacteriaceae and nonfermentative bacteria, and the data are shown in Fig. 3.

\section{Analysis of major risk factors}

Analysis of major risk factors was performed according to the literature [7]. The risk factors for common MDRO infections are currently believed to be age, invasive operation, treatment with three or more antimicrobial agents, and previous multiple or long-term hospitalization. In this study, univariate analyses of 15 common potential risk factors were conducted, and the results are shown in Table 1. Multivariate logistic regression analysis was performed for 7 types of risk factors, and the results suggested that the use of third- or fourth-generation cephalosporins was an independent risk factor for Gramnegative MDRO infections in patients with AP $(P<0.05)$, as shown in Table 2.

\section{Discussion}

$\mathrm{AP}$ is a common disease of the digestive system with different clinical manifestations. Especially in SAP patients with a poor prognosis and a fatality rate as high as $30 \% \sim 40 \%$ due to complications, infection frequently occurs and is partly caused by pathogens that pass through biliary system. Although there are many methods to treat corresponding complications, the effect is not totally satisfactory [8]. Therefore, it is necessary to actively prevent pathogen infection in AP and avoid SAP, and important to understand the characteristics of pathogen infections and their associated risk factors [9]. At 


\section{A}

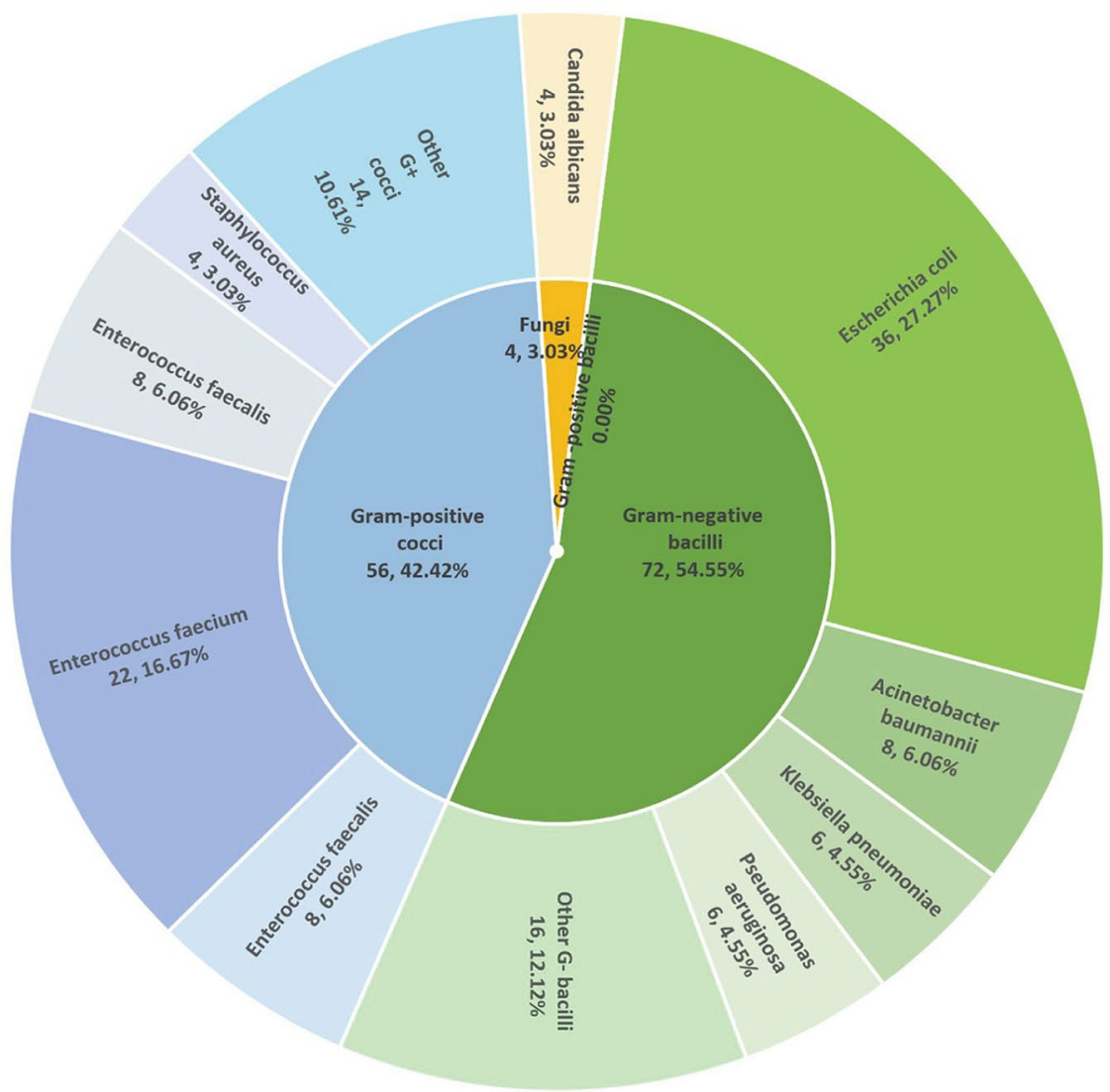

$\mathbf{B}$

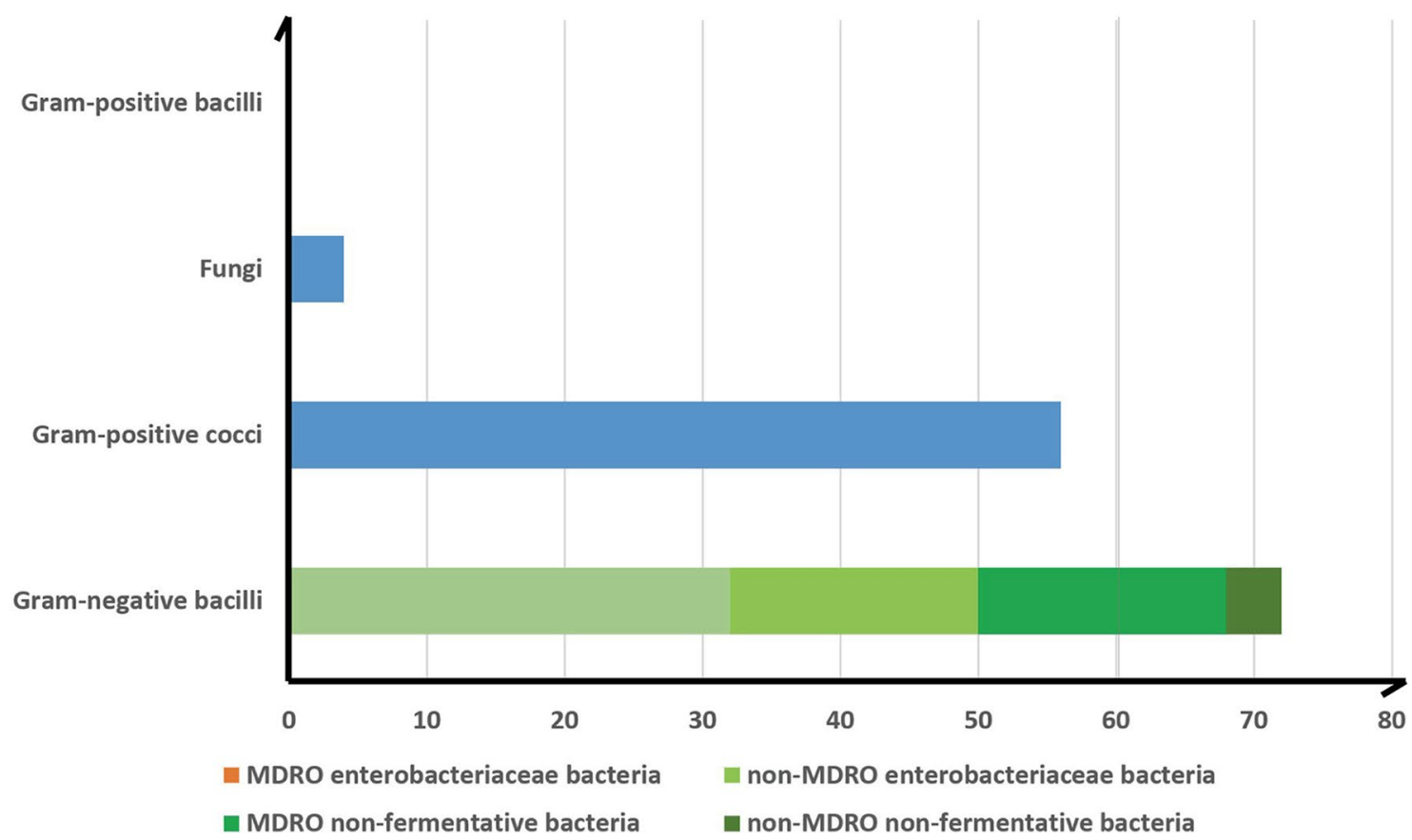

Fig. 1 The distribution and proportion of pathogenic bacteria in AP patients. A The distribution and proportion of pathogenic bacteria in 120 cases AP patients. B The distribution and proportion of pathogenic bacteria in 120 cases AP patients by dividing of MDRO, Enterobacteriaceae and Non-fermenter bacteria 

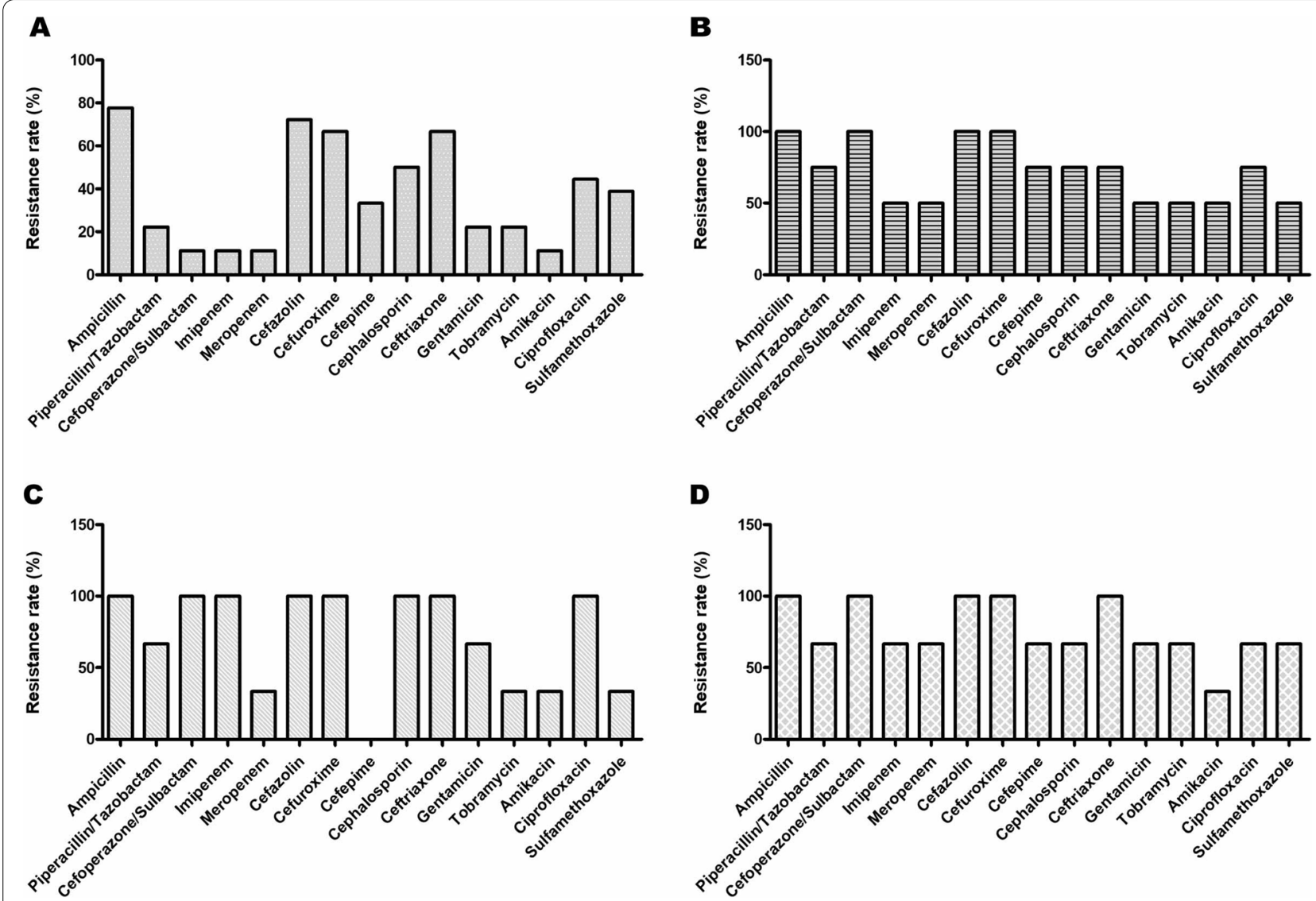

Fig. 2 Drug resistance analysis of main Gram-negative pathogenic bacteria in AP patients. A Drug resistance analysis of Escherichia coli. B Drug resistance analysis of Acinetobacter baumannii. C Drug resistance analysis of Klebsiella pneumoniae. D Drug resistance analysis of Pseudomonas aeruginosa
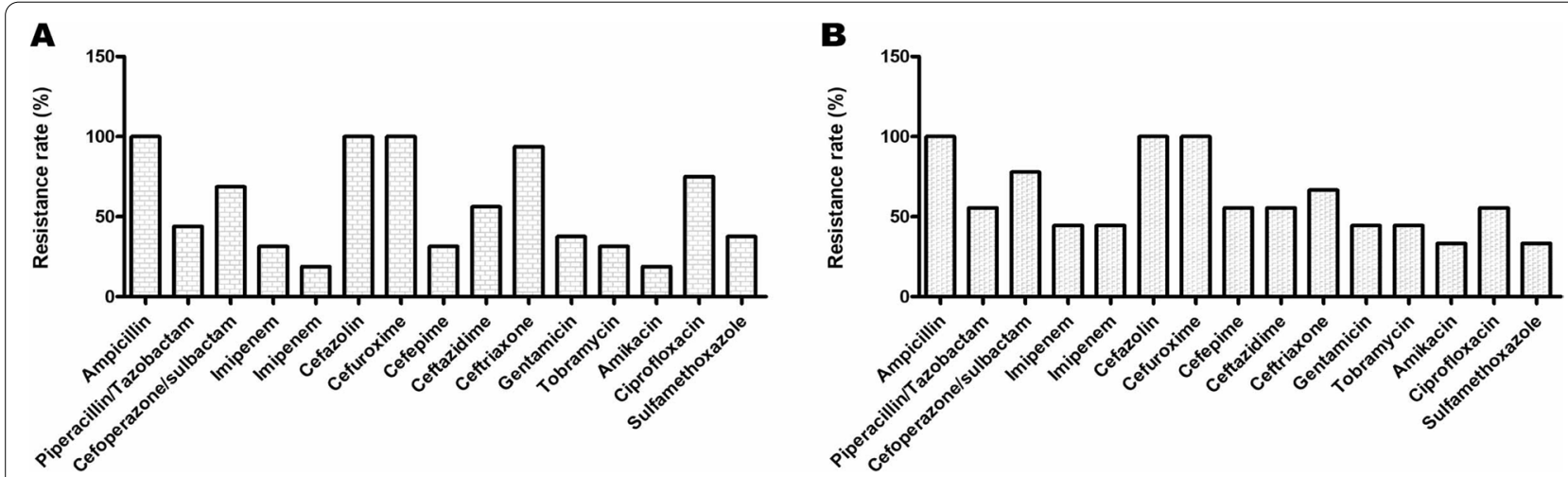

Fig. 3 Analysis of drug resistance rates of biliary enteroderaceae and non-fermentative bacteria MDRO in AP patients. A Drug resistance analysis of Gram-negative enterobacteriaceae MDRO. B Drug resistance analysis of Gram-negative non-fermentation MDRO

present, there are many scientific studies on AP [10], but unfortunately there are relatively few and controversial studies on pathogen infections [11], hence, it is necessary to conduct in-depth research on infectious pathogens in bile of AP patients.

In this study, the bile samples were incubated and separated by a BacT/Alert3D automated blood culture 
Table 1 Single factor analysis of risk factors for bile Gram - negative MDRO infection in AP patients

\begin{tabular}{|c|c|c|c|c|}
\hline Dangerous factor & MDRO group $(n=50)$ & $\begin{array}{l}\text { Non-MDRO group } \\
(n=22)\end{array}$ & $\begin{array}{l}t \text { value } / Z \text { value } / X^{2} \\
\text { value }\end{array}$ & $P$ value \\
\hline Age (Years) & $60.62 \pm 14.01$ & $65.70 \pm 18.22$ & $0.8969^{\mathrm{a}}$ & 0.3761 \\
\hline Hospital stays (Day) & $35.65 \pm 26.58$ & $15.26 \pm 14.25$ & $-3.211^{b}$ & $<0.001$ \\
\hline Duration of antimicrobial use (Day) & $28.44 \pm 25.41$ & $12.47 \pm 10.98$ & $-4.545^{c}$ & $<0.001$ \\
\hline Types of antimicrobial agents used (Type) & $4.58 \pm 3.22$ & $2.40 \pm 1.57$ & $-4.106^{d}$ & $<0.001$ \\
\hline Gender (Male) & 30 & 10 & $0.954^{e}$ & 0.791 \\
\hline Use of third or fourth generation Cephalosporins & 44 & 8 & $0.881^{f}$ & $<0.001$ \\
\hline Use of Carbapenems & 48 & 6 & $21.65^{9}$ & $<0.001$ \\
\hline Invasive operation & 40 & 6 & $1.050^{h}$ & $<0.001$ \\
\hline Ventilation & 6 & 2 & $1.352^{i}$ & 0.066 \\
\hline Operation & 30 & 10 & $0.592^{j}$ & 0.541 \\
\hline Combined with biliary tract disease & 10 & 8 & $15.472^{k}$ & $<0.001$ \\
\hline Associated abdominal infection & 20 & 6 & $0.683^{1}$ & 0.539 \\
\hline Combined diabetes & 10 & 4 & $0.852^{m}$ & 0.594 \\
\hline Complicated with malignant tumor & 0 & 0 & $3.246^{n}$ & 0.800 \\
\hline Combined with COPD or respiratory failure & 4 & 0 & $4.615^{\circ}$ & 0.763 \\
\hline
\end{tabular}

${ }^{a}$ was $t$ value of T test, ${ }^{b-d}$ were $Z$ value of Kruskal-Wallis Test, respectively, ${ }^{e-o}$ were $x^{2}$ value

Table 2 Multivariate logistic regression analysis of bile Gram - negative MDRO infection in AP patients

\begin{tabular}{llllllr}
\hline Risk factors & $\begin{array}{l}\text { Partial regression } \\
\text { coefficient }\end{array}$ & Standard error & Wald & P value & OR value & 95\% Cl \\
\hline Hospital stays & -0.018 & 0.014 & 0.987 & 0.594 & 1.172 & $0.865-1.479$ \\
Duration of antimicrobial use & 0.034 & 0.144 & 1.002 & 0.951 & 1.095 & $0.921-1.268$ \\
Types of antimicrobial agents used & 0.411 & 0.197 & 1.905 & 0.220 & 1.112 & $0.819-1.405$ \\
Third or fourth generation cephalosporins & 0.957 & 0.157 & 2.456 & 0.040 & 2.120 & $0.955-3.287$ \\
Carbapenems & 0.885 & 0.386 & 0.566 & 0.112 & 1.579 & $0.967-2.191$ \\
Invasive operation & -0.157 & 0.234 & 0.020 & 0.812 & 1.562 & $0.657-2.468$ \\
Combined with biliary tract disease & 0.592 & 0.805 & 0.244 & 0.620 & 1.364 & $0.247-2.482$ \\
\hline
\end{tabular}

instrument [12] in accordance with international standard operation procedures for bacterial detection [13, 14]; this method can guarantee the growth and accurate identification of specific organisms, even if the samples contained multiple species [13]. According to the data obtained from bile specimens from 560 AP patients in hospitals, 120 positive cases and 132 strains of pathogenic bacteria were identified, with a positive infection rate of $21.43 \%$, which was lower than that obtained in an analysis without stratification by sample type [15], similar to those in blood culture specimens in some hospitals in northern China without disease classification [16], and higher than those in blood culture specimens in some hospitals in central cities of China [17]. The percentage of specimens with more than one pathogen was 9.1\%, which was lower than the proportion of blood culture specimens [18]. Here, the main pathogenic bacteria detected in bile were Escherichia coli (36 strains), Enterococcus faecium (22 strains), Acinetobacter baumannii
(8 strains), Enterococcus faecalis (8 strains), coagulasenegative Staphylococcus (8 strains) and Klebsiella pneumoniae (6 strains). Gram-negative bacteria were mainly detected (72 cases, accounting for $54.55 \%$ ), among which Escherichia coli, Acinetobacter baumannii, Klebsiella pneumoniae and Pseudomonas aeruginosa were the most abundant. It has been reported in the literature that bacteria in blood culture samples are mainly Gram-positive bacteria [19], and the second most abundant bacterial species are Escherichia coli and Klebsiella pneumoniae $[17,20]$; this was not consistent with our results. Among the bile pathogens in AP patients in our study, Escherichia coli had a high detection rate.

Detection of pathogen resistance is an important reference for guiding clinically rational drug use; thus, we analyzed the drug resistance of the top four Gram-negative bacilli bacteria. Extended-spectrum beta-lactamase (ESBL)-producing Escherichia coli was $66.67 \%$ resistant to ampicillin, cefazolin and cefuroxime. The rates of 
cephalosporin and ceftriaxone resistance were both more than $50.00 \%$, and the rate of ciprofloxacin resistance was greater than $40.00 \%$, the less resistance to amikacins was detected. The drug sensitivity was lower than that reported in the literature for blood samples [21]; the reasons for this require further research.

Acinetobacter baumannii is an important pathogen that causes nosocomial infections in China and worldwide [22]. It is intrinsically resistant to ampicillin, amoxicillin, amoxicillin/clavulanic acid, aztreonam, ertapenem, first-generation cephalosporins, second-generation cephalosporins and fosfomycin. Although in vitro sensitivity experiments showed good results, the clinical application of these agents should not be carried out because of antimicrobial therapy failure [23]. Bacterial infection and drug resistance rates have shown increasing trends. Our study showed that Acinetobacter baumannii had high drug resistance and a tendency towards multidrug resistance, similar to the results of previous literature reports $[18,23]$. In addition, the resistance rate for hydrocarbon enzyme alkenes was more than $50.00 \%$, which was slightly higher than that in hospital data reported in China [24] and lower than that in some hospital reports from China [23]. The conflicting results may be due to the clinically widespread use of these drugs, the time of administration, the small number of specimens collected in this study, or other factors, such as regional differences, the specific reasons remain to be further explored. However, the results indicate that drug resistance in $\mathrm{Aci}$ netobacter baumannii is becoming increasingly serious. Since conventional disinfection methods inhibit but do not kill Acinetobacter baumannii, patients with weak immunity or trauma may be more susceptible to infection caused by bacteria present on the hands of clinical staff or on medical devices that are not thoroughly sterilized [25]. Therefore, the prevention and treatment of Acinetobacter baumannii infection should not be disregarded, and secondary infections should be closely monitored during hospital treatment and care, especially in AP patients.

The detection rate of ESBL-producing Klebsiella pneumoniae was $100.00 \%$, similar to the results of other reports [26]. Resistance to cefuroxime, cefazolin, ceftazidime, ceftriaxone, ciprofloxacin, cefoperazone/ sulbactam and imipenem reached $100.00 \%$. Slight sensitivity to amikacin was detected, and the resistance was higher than that in bacteria isolated from the Intensive care unit (ICU) [27]. Similarly, Pseudomonas aeruginosa had $66.67 \%$ resistance to carbapenems, while it was only mildly sensitive to amikacin. The high drug resistance rate in both of these pathogens may be related to the statistical deviation caused by the number of patients enrolled, or it may be that drug resistance situation among pathogenic bacteria in the bile of AP patients is serious.

With the extensive use of antimicrobial agents in recent years, MDROs have become important pathogens of nosocomial infections. MDROs belonging to Enterobacteriaceae and nonfermentative MDROs were analyzed separately. MDROs are resistant to ampicillin, first-generation cephalosporins and second-generation cephalosporins. Moreover, the sensitivity trends of ceftazidime, piperacillin/tazobactam, cefoperazone/sulbactam, gentamicin and tobramycin were similar. However, resistance to both ceftriaxone and ciprofloxacin was higher than that to ciprofloxacin. In contrast, resistance to aminoglycosides, cefepime and carbapenems were higher. It was concluded that the drug resistance patterns of MDROs belonging to Enterobacteriaceae and nonfermentative MDROs were very different, which is not conducive to clinical anti-infection treatment in AP patients.

Analysis of resistance of MDROs and risk factors for Gram-negative MDRO infection can help identify relevant risk factors and prevent infection [28]. Our results showed that seven factors, such as the length of hospital stay, time, antibacterial drug use, antimicrobial type, use of third- or fourth-generation cephalosporins, use of penicillium carbon alkene antimicrobial agents, invasive operations, and complication of biliary tract disease, are risk factors for Gram-negative MDRO infection. Multivariable logistic regression analysis of these factors indicated that the use of third- or fourth-generation cephalosporins in AP patients with the presence of biliary Gram-negative MDROs was an independent risk factor for infection and high drug resistance. Therefore, the rational use of third- or fourth-generation cephalosporins is an important measure in preventing MDRO infection.

\section{Conclusions}

In summary, for AP patients, the result detected in bile mainly were Gram-negative bacteria, and the main pathogenic bacteria detected in descending order were Escherichia coli, Enterococcus faecium, Acinetobacter baumannii, Enterococcus faecalis, coagulase-negative Staphylococcus and Klebsiella pneumoniae. Moreover the use of third- or fourth-generation cephalosporins of biliary Gram-negative MDROs was an independent risk factor for infection and high drug resistance. Therefore hospitals should focus on strengthening the management, prevention and control of MDRO infections.

\section{Methods}

\section{Study subjects}

The study recruited 560 patients hospitalized from January 2016 to December 2020. There were 304 male and 256 female patients. All the subjects were aged from 19 
to 80 years, with an average age of $57.5 \pm 25$ years, and were firstly diagnosed with AP. Among them, 8 patients (1.43\%) had severe AP as the single diagnosis, 186 patients $(33.21 \%)$ of moderately severe AP had a secondary diagnosis of biliary disease, 82 patients (14.64\%) of moderately severe AP had a secondary diagnosis of abdominal infection, and the remaining 284 patients (50.71\%) had only AP.

The clinical specimens were collected according to the revised 2012 Atlanta Classification [29]. According to the revised 2012 Atlanta Classification, the diagnosis of AP requires the presence of 2 of the following: abdominal pain characteristic of AP; serum amylase and/or lipase activity at least 3 times higher than the referent limit; and AP signs on abdominal computed tomography or transabdominal ultrasonography. In addition, AP can be classified into two types. In this study, AP was defined as the absence of organ failure and local complications. SAP was defined as the presence of local complications and/ or transient or persistent organ failure and / or exacerbations of comorbidities. AP patients, who were identified by either the International Classification of Diseases, 10th revision, (ICD - 10) code K85 or the ICD - 9 CM code 577.0, were selected.

\section{Sample collection}

Specimens were collected when the AP patients had clinically biliary sampling symptoms or infectious symptoms of biliary tract, and no antibiotics had been used. Bacterial culture identification was performed; $2 \sim 10 \mathrm{ml}$ of bile was extracted via gallbladder or bile duct puncture and drainage.

\section{Sample culture and bacterial detection}

The bile samples were aseptically injected into aerobic and anaerobic culture bottles, mixed well, placed in a BacT/Alert3D automated blood culture instrument, and continuously monitored for 2 days. Cultures with no positive indication at the end of the process were transferred to blood agar medium for culture and judged negative if no bacteria were detected and positive when the bacteria had proliferated and the instrument detected a yellow color at a certain time. The positive bile samples in the culture bottles were transferred to blood agar medium, Sabouraud fungi medium, McConkey agar medium and chocolate agar medium (Tianjin Jinzhang Biotechnology Development Co., Ltd. Tianjin, China.), which were then placed into 371 carbon dioxide bacteria incubators at $35^{\circ} \mathrm{C}$ and $5 \% \mathrm{CO}_{2}$ for $18 \sim 24 \mathrm{~h}$. If necessary, anaerobic culture bags (1.5l anaerobic culture bags; Qingdao Haibo Biotechnology, Shandong, China) were used for further culture and separation.

\section{Bacterial strain identification and drug susceptibility testing}

When the bacteria were obtained, they were manually sampled with a VITEK 2 compact automatic microbial analysis system. Strain identification and drug susceptibility profiles were identified by the VITEK 2 compact automatic microbial analysis system. The results of the drug susceptibility tests were analyzed and compared with the standard interpretation of the Clinical Laboratory Standardization Institute (CLSI) 2015-2019. All testing operations were conducted in strict accordance with the national clinical testing operating procedures.

\section{Standard quality control}

Standard control strains, namely, Staphylococcus aureus ATCC29213, Escherichia coli ATCC25922 and Enterobacter cloacae ATCC700323 (provided by the clinical testing center of the National Health and Family Planning Commission in China), were tested in parallel.

\section{Independent risk factors for death were identified with as multivariate Cox regression model}

Seventy-two cases with Gram-negative bacilli isolation during hospitalization from 2016 to 2020 were divided into a multidrug-resistant bacteria group (50 cases) and a non-multidrug-resistant bacteria group (22 cases), and their clinical characteristics were retrospectively analyzed to identify potential risk factors. Independent risk factors for death were identified with as multivariate Cox regression model.

\footnotetext{
Abbreviations

AP: Acute pancreatitis; SAP: Severe acute pancreatitis; MDRO: Multidrug-resistant bacteria; non-MDRO: Non-multidrug-resistant bacteria; ESBL: Extendedspectrum beta-lactamase; ICU: Intensive care unit; ICD - 9 CM: International Classification of Diseases, Clinical Modification, 9th revision; CLSI: Clinical Laboratory Standardization Institute; ORs: Odds ratios; Cls: Confidence intervals.
}

\section{Acknowledgments}

We thank all our colleagues from the Department of Laboratory Science at Tianjin Medical University NanKai Hospital, who collaborated in this study.

\section{Authors' contributions}

SC, YC and XG conceived and designed the study. SC, JS, MC, JM, ZZ and RW performed the experiments. SC wrote the paper. All authors read and approved the final manuscript.

\section{Funding}

This work was supported by grant 81703846 from the National Natural Science Foundation of China (NSFC). Shayan Chen was a recipient of the First Batch of Young Medical Professionals of Tianjin Health Commission in 2018.

\section{Availability of data and materials}

The datasets analyzed during the current study are available from the first author upon reasonable request. 


\section{Declarations}

\section{Ethics approval and consent to participate}

The present study was approved (No. NKYY_YX_IRB_2018_037_01) by the Medical Ethics Committee of Tianjin Medical University, NanKai Hospital. Because the study was retrospective in nature, informed consent was not required. All patient data were anonymized and are not available to the public. All methods were performed in accordance with the relevant guidelines and regulations of national clinical testing operating procedures in China.

\section{Consent for publication}

Not applicable.

\section{Competing interests}

No potential conflicts of interest are disclosed.

\section{Author details}

'Department of Laboratory Science, Tianjin Medical University NanKai Hospital, Tianjin 300100, China. ${ }^{2}$ Tianjin Key Laboratory of Acute Abdomen Disease Associated Organ Injury and ITCWM Repair, Tianjin Medical University NanKai Hospital, Tianjin 300100, China. ${ }^{3}$ Tianjin Medical University, Tianjin 300070, China. ${ }^{4}$ Department of Surgery, Tianjin Medical University NanKai Hospital, 122 Sanwei Road, Nankai District, Tianjin 300100, China. ${ }^{5}$ Binzhou Medical University, Yantai City 264003, Shandong, China.

Received: 8 February 2021 Accepted: 28 September 2021

Published online: 05 October 2021

\section{References}

1. Boxhoorn L, Voermans RP, Bouwense SA, Bruno MJ, Verdonk RC, Boermeester MA, et al. Acute pancreatitis. Lancet. 2020;396:726-34.

2. Furuya T, Soeno T, Komatsu M. Strategy for bacterial translocation in acute pancreatitis. Nihon Shokakibyo Gakkai Zasshi. 2004;101:502-9.

3. Mandryka Y, Klimczak J, Duszewski M, Kondras M, Modzelewski B. Bile duct infections as a late complication after endoscopic sphincterotomy. Pol Merkur Lekarski. 2006;21:525-7.

4. Yangzom T, Tsering DC, Kar S, Kapil J. Antimicrobial susceptibility trends among pathogens isolated from blood: a 6-year retrospective study from a tertiary care hospital in East Sikkim. India J Lab Physicians. 2020;12(01):3-9.

5. Wang J, Zhang H, Yan J, Zhang T. Literature review on the distribution characteristics and antimicrobial resistance of bacterial pathogens in neonatal sepsis. J Matern Fetal Neonatal Med. 2020:1-10. https://doi.org/10.1080/ 14767058.2020.1732342.

6. Chen Y, Suo J, Du M, Chen L, Liu Y, Wang L, et al. Clinical features, outcomes, and risk factors of bloodstream infections due to Stenotrophomonas maltophilia in a tertiary-care hospital of China: a retrospective analysis. Biomed Res Int. 2019:4931501. https://doi.org/10.1155/2019/4931501.

7. Lin J, He SS, Xu YZ, Li HY, Wu XM, Feng JX. Bacterial etiology in early readmission patients with acute exacerbation of chronic obstructive pulmonary disease. Afr Health Sci. 2019;19:2073-81.

8. Lankisch PG, Apte M, Banks PA. Acute pancreatitis. Lancet. 2015. https://doi. org/10.1016/S0140-6736(1014)60649-60648.

9. Tsui NC, Zhao E, Li Z. Microbiological findings in secondary infection of severe acute pancreatitis: a retrospective clinical study. Pancreas. 2009:38:499-502.

10. Van Dljk SM, Hallensleben ND, van Santvoort HC, Fockens P, van Goor H, Bruno MJ, et al. Acute pancreatitis: recent advances through randomised trials. Gut. 2017;66:2024-32.

11. Wolbrink DRJ, Kolwijck E, Ten Oever J, Horvath KD, Bouwense SAW, Schouten JA. Management of infected pancreatic necrosis in the intensive care unit: a narrative review. Clin Microbiol Infect. 2020;26:18-25.

12. Thorpe TC, Wilson ML, Turner JE, DiGuiseppi JL, Willert M, Mirrett S. BacT/ ALERT: an automated colorimetric microbial detection system. J Clin Microbiol. 1990;28:1608-12

13. CLSI. Quality control for commercially prepared microbiological culture media; approved standard —-third edition. CLSI/NCCLS document; 2004. p. M22-A23
14. She RC, Romney MG, Jang W, Walker T, Karichu JK, Richter SS. Performance of the BacT/Alert Virtuo Microbial Detection System for the culture of sterile body fluids: prospective multicentre study. Clin Microbiol Infect. 2018;24:992-6.

15. Fan N, Hu Y, Shen H, Liu S, Zhao G, Sun L, et al. Compositional and drugresistance profiling of pathogens in patients with severe acute pancreatitis: a retrospective study. BMC Gastroenterol. 2020;20:405. https://doi.org/10. 1186/s12876-020-01563-X

16. MacFadden DR, Coburn B, Shah N, Robicsek A, Savage R, Elligsen M, et al. Utility of prior cultures in predicting antibiotic resistance of bloodstream infections due to Gram-negative pathogens: a multicentre observational cohort study. Clin Microbiol Infect. 2018;24:493-9.

17. Hayakawa K, Mezaki K, Kobayakawa M, Yamamoto K, Mutoh Y, Tsuboi M, et al. Impact of rapid identification of positive blood cultures using the Verigene system on antibiotic prescriptions: a prospective study of communityonset bacteremia in a tertiary hospital in Japan. PLoS One. 2017. https://doi. org/10.1371/journal.pone.0181548

18. Tian H, Chen L, Wu X, Li F, Ma Y, Cai Y, et al. Infectious complications in severe acute pancreatitis: pathogens, drug resistance, and status of nosocomial infection in a university-affiliated teaching hospital. Dig Dis Sci. 2020;65:2079-88.

19. Mowbray NG, Ben-Ismaeil B, Hammoda M, Shingler G, Al-Sarireh B. The microbiology of infected pancreatic necrosis. Hepatobiliary Pancreat Dis Int. 2018:17:456-60

20. Droz N, Hsia Y, Ellis S, Dramowski A, Sharland M, Basmaci R. Bacterial pathogens and resistance causing community acquired paediatric bloodstream infections in low- and middle-income countries: a systematic review and meta-analysis. Antimicrob Resist Infect Control. 2019. https://doi.org/10. 1186/s13756-019-0673-5.

21. Wang E, Zhang C, Tang Y, Liang T, Deng Y, Li X, et al. Antibiotic susceptibility and prognostic analysis of Gram negative bacterial bloodstream infection in patients with acute leukemia. Zhong Nan Da Xue Xue Bao Yi Xue Ban. 2020:45:1068-73.

22. Lai CC, Chen CC, Lu YC, Chuang YC, Tang HJ, et al. In vitro activity of cefoperazone and cefoperazone-sulbactam against carbapenem-resistant Acinetobacter baumannii and Pseudomonas aeruginosa. Infect Drug Resist. 2019;12:25-9.

23. Boral B, Unaldi Ö, Ergin A, Durmaz R, Eser ÖK, Acinetobacter Study Group. A prospective multicenter study on the evaluation of antimicrobial resistance and molecular epidemiology of multidrug-resistant Acinetobacter baumannii infections in intensive care units with clinical and environmental features. Ann Clin Microbiol Antimicrob. 2019. https://doi.org/10.1186/ s12941-019-0319-8.

24. Rezaei A, Fazeli H, Faghri J. Investigation of carbapenem resistant Acinetobacter baumannii ST2 in Iran. Acta Microbiol Immunol Hung. 2020. https:// doi.org/10.1556/030.2020.01164.

25. Selvaraj A, Muthuramalingam P, Sethupathy S, Subramenium GA, Ramesh M, Pandian SK. Proteomic and systematic functional profiling unveils citral targeting antibiotic resistance, antioxidant defense, and biofilm-associated two-component systems of Acinetobacter baumannii to encumber biofilm and virulence traits. 2020. https://doi.org/10.1128/mSystems.00986-20.

26. Tugal D, Lynch M, Hujer AM, Rudin S, Perez F, Bonomo RA. Multi-drugresistant Klebsiella pneumoniae pancreatitis: a new challenge in a serious surgical infection. Surg Infect. 2015;16:188-93.

27. Zhang Y, Zhao C, Wang Q, Wang X, Chen H, Li H, et al. High prevalence of hypervirulent Klebsiella pneumoniae infection in China: geographic distribution, clinical characteristics, and antimicrobial resistance. Antimicrob Agents Chemother. 2016;60:6115-20.

28. Soares FS, Amaral FC, Silva NLC, Valente MR, Santos LKR, Yamashiro LH, et al. Antibiotic-induced pathobiont dissemination accelerates mortality in severe experimental pancreatitis. Front Immunol. 2017;8:1890.

29. Banks PA, Bollen TL, Dervenis C. Classification of acute pancreatitis--2012: revision of the Atlanta classification and definitions by international consensus. Gut. 2013;62:102-11.

\section{Publisher's Note}

Springer Nature remains neutral with regard to jurisdictional claims in published maps and institutional affiliations. 